\title{
An inter-frame dynamic double-threshold energy detection for spectrum sensing in cognitive radios
}

\author{
Caimei Fu' ${ }^{1}$, Youming $\mathrm{Li}^{1 *}$, Yucheng He${ }^{2}$, Ming Jin' ${ }^{1}$, Gang Wang ${ }^{1}$ and Peng Lei ${ }^{1}$
}

\begin{abstract}
This paper proposes an inter-frame dynamic double threshold (IF-DDT) spectrum sensing algorithm in order to improve the sensing performance based on energy detection (ED) in cognitive radios (CRs). Based on both the activity model of the primary user (PU) and the sensing mechanism of the secondary user (SU), the proposed algorithm exploits the relationship between two adjacent sensing frames and designs dynamic double thresholds for each sensing frame to enhance spectrum sensing performance when the received energy cannot give a reliable local decision. The detection probability and false alarm probability of the proposed sensing scheme are analyzed, and an algorithm for searching the optimal dynamic double thresholds is derived with very low complexity according to the Neyman-Pearson (NP) test criterion. Theoretical analysis and simulation results show that the proposed algorithm outperforms the ED algorithm.
\end{abstract}

Keywords: Cognitive radios, Dynamic double thresholds, Energy detection, Inter-frame spectrum sensing

\section{Introduction}

Nowadays, wireless communications have been experiencing an increasing tension of the frequency resource which, however, is far from fully utilized under the static spectrum assignment policy [1]. Cognitive radio (CR) technology provides a promising solution to the conflict by allowing the second user (SU) to opportunistically access the licensed spectrum band when it is temporarily not occupied by the primary user (PU) [2].

Spectrum sensing is a critical aspect of CR systems that aims to identify the working state of the PU (i.e., ON or OFF, indicating whether the licensed spectrum is occupied or not, respectively) before allowing the SU temporarily access the channel without causing harmful interference to the PU. Typically, the SU operates spectrum sensing frame by frame, and each frame is divided into two parts: the sensing duration and the data duration [3-5]. The SU detects whether the licensed spectrum is accessed within the sensing duration, and then transmits

*Correspondence: liyouming@nbu.edu.cn

${ }^{1}$ College of Information Science and Engineering, Ningbo University, Ningbo, China

Full list of author information is available at the end of the article data within the data duration if the sensing result is OFF (which identifies that the PU's state is OFF); otherwise, the SU keeps silent.

The accuracy of spectrum sensing is measured by two probabilities: the false alarm probability (denoted as $P_{f}$ ) and the detection probability (denoted as $P_{d}$ ). The false alarm probability is defined as the probability that the PU's state is identified as ON when its real state is OFF, while the detection probability is defined as the probability that the PU's state is identified as ON when its real state is also ON. Clearly, a low false alarm probability improves the efficiency of the unused spectrum, whereas a high detection probability reduces the resulting interference in the licensed users $[4,6]$.

A number of spectrum sensing methods have been proposed for CR systems, such as energy detection (ED), cyclostationarity feature detection, second-order statistics detection, matched-filtering detection, compressive sensing detection $[7,8]$, and multiple antenna detection [9]. The energy detection has been preferred due to its feasible applicability and low implementation complexity. The cyclostationarity feature detection differentiates noise from PU signals by exploiting the cyclostationarity features of the received signals. However, it has high 
computational complexity and requires the knowledge of the cyclic frequencies which is difficult to be obtained. The second-order statistics-based detection is subject to the modulations of primary signals. The matchedfiltering detection is the optimum method only when perfect information about the waveforms of the PU is given. The compressive sensing detection makes use of the sparse structure of primary signals. The multiple antenna detection effectively overcomes the noise uncertainty and improves the detection performance based on the spatial correlation in the multi-antenna receiver. Nevertheless, it requires the SU equipped with multi-antenna receivers.

In order to avoid error detection caused by dramatic energy decrease, the average signal power of several past sensing frames is used in [10] for the ED schemes. However, conventional ED-based spectrum sensing schemes usually employ only a single fixed threshold. When the received energy of the SU nears the threshold, the decision for the PU's state is subject to a high probability of misjudgment, thus yielding a loss in sensing performance. In order to alleviate this problem, the doublethreshold energy detection method is discussed in [11]. This method can decrease the interference of the SU to the PU. However, how to set the two thresholds and deal with the received energy falling between the two thresholds are not discussed. Double-threshold energy detection method is also discussed in cooperative spectrum sensing environment [12]. In this method, each SU sends its observed energy to the fusion center (FC) when the energy falls between the two thresholds; otherwise, the $\mathrm{SU}$ sends its decision results. The final decision is made based on the soft combination of the received energy and hard combination of the received decision results in the FC. Therefore, the performance improvements of cooperative spectrum sensing schemes are obtained at the cost of extra control messages and increased computation complexities [13].

An alternative way of improving the ED schemes is to take into account the PU's activity model during the sensing process. A model of two-state Markov chain is used as an adequate mean accurately describing spectrum occupancy in the time domain in [14-17]. On the one hand, the durations of the PU's states (ON and OFF) are respectively exponentially distributed $[18,19]$. On the other hand, the frame length of the SU is much shorter than both the average durations of the PU's ON and OFF states $[20,21]$, which implies that the PU's activities over successive sensing frames are not independent, especially between adjacent frames. Furthermore, the PU stays at the same state with a high probability during the whole sensing frame [22]. These properties can be exploited to improve the sensing performance of the ED schemes.
In [22], the received energy sequence over successive sensing frames is modeled as a continuous hidden Markov chain, and the final decision is made according to the combined observations from all previous sensing frames. However, this scheme needs to perform simultaneously spectrum sensing and data transmission and hence suffers from implementation difficulties due to self-interference [23]. In [24] and [25], the PU activity is modeled as a Markov chain, and the final decision is also based on history observations but using different combining rules from that of [22]. However, in the schemes in [24] and [25], each sensing frame is further divided into many short slots for either dynamic spectrum sensing or data transmission. This frequent sensing-transmitting alternating strategy may cause synchronization difficulty on the receiving terminal.

In this paper, using a two-state Markov chain model of the PU's activity, an inter-frame dynamic double threshold (IF-DDT) ED scheme is proposed. Firstly, the relationship between two adjacent sensing frames is analyzed, and the IF-DDT scheme for each sensing frame is designed based on dynamic double thresholds. Then, according to the Neyman-Pearson (NP) test criterion, an optimization problem is formulated to acquire the optimal dynamic double thresholds which maximize the detection probability while maintaining a maximum tolerable false alarm probability. Finally, the optimization problem is transformed into an easily solvable problem and an algorithm for searching the optimal dynamic double thresholds are derived with very low complexity. Theoretical analysis and computer simulations show that the proposed IF-DDT scheme can achieve performance improvement due to dynamic double thresholds even when the received energy cannot give a reliable local decision. Compared to reference [11], theoretical analysis and the implementation method are provided for the proposed double-threshold energy detection scheme.

The remainder of this paper is organized as follows. In Section 2, the system model is presented. In Section 3, the IF-DDT scheme is proposed, and the optimal dynamic double thresholds are derived. Section 4 evaluates the proposed scheme for spectrum sensing through simulations. Finally, Section 5 concludes the paper.

\section{System model}

We consider a CR system with one PU, one SU, and a single channel licensed to the PU. The PU operates between $\mathrm{ON}$ and OFF states alternately, while the SU executes spectrum sensing to the licensed channel and opportunistically transmits data in a frame-wise manner. In the sequel, the PU's ON and OFF states are represented by integers "1" and "0," respectively. The system model is presented in Fig. 1 , which shows the PU's activity model and the SU's sensing mechanism. 
PU

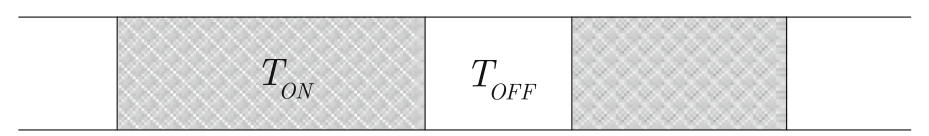

SU

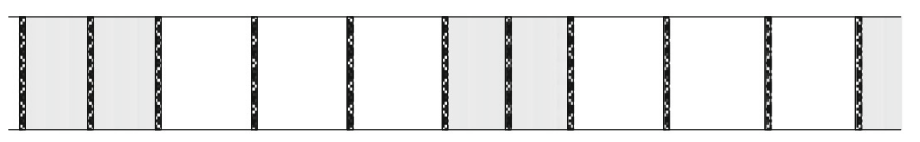

$\mathrm{PU} \square \mathrm{ON}$

OFF

SU sensing duration $\tau$

data duration $\left(T F_{k}-\tau\right)$

silence duration $\left(T F_{k}-\tau\right)$

Fig. 1 The PU's activity and the SU's sensing mechanism

\subsection{The PU's activity model and the SU's sensing mechanism}

Let $T_{1}$ and $T_{0}$ denote the durations that the PU stays respectively in ON and OFF states as shown in Fig. 1. It is well accepted that the durations are exponentially distributed with expectations $\bar{T}_{1}=\alpha$ and $\bar{T}_{0}=\beta$ [25]. The PU's average activity period is defined as $\bar{T}_{P U}=\bar{T}_{1}+\bar{T}_{0}$, and the PU's traffic load is defined as $\eta_{\mathrm{PU}}=\bar{T}_{1} / \bar{T}_{\mathrm{PU}}$.

We assume that the SU allocates each frame $k$ with a dynamic length $T F_{k}$, consisting of fixed sensing duration $\tau$ and varying data transmission duration $T F_{k}-\tau$ for $k=$ $1,2, \ldots$. Furthermore, we assume that the lengths satisfy the conditions that $\tau \ll T F_{k}, T F_{k} \ll \bar{T}_{1}$, and $T F_{k} \ll \bar{T}_{0}$.

Let $z_{k} \in\{0,1\}$ denote the real state of the PU within the sensing duration of any specific frame $k$ at the SU. On the above assumptions, it is clear that there is a high probability that $z_{k}$ remains unchanged within a whole frame and that $z_{k}$ is closely related to the past state $z_{k-1}$ with respect to the preceding frame $k-1$ for $k>1[18,20,26]$.

The PU's activity can be modeled as a two-state discretetime Markov chain with the stationary distribution

$$
P\left\{z_{k}=0\right\}=\frac{\alpha}{\alpha+\beta}, \quad P\left\{z_{k}=1\right\}=\frac{\beta}{\alpha+\beta},
$$

and the transition matrix

$$
\begin{aligned}
\mathbf{P}_{k-1} & =\left[\begin{array}{ll}
p_{00, k-1} & p_{01, k-1} \\
p_{10, k-1} & p_{11, k-1}
\end{array}\right] \\
& =\frac{1}{\alpha+\beta}\left[\begin{array}{ll}
\alpha+\beta e^{-(\alpha+\beta) T F_{k-1}} & \beta-\beta e^{-(\alpha+\beta) T F_{k-1}} \\
\alpha-\alpha e^{-(\alpha+\beta) T F_{k-1}} & \beta+\alpha e^{-(\alpha+\beta) T F_{k-1}}
\end{array}\right]
\end{aligned}
$$

where $p_{i j, k-1} \triangleq P\left\{z_{k}=j \mid z_{k-1}=i\right\}$ denotes the state transition probability over two adjacent frames $k-1$ and $k$ for $i, j \in\{0,1\}$; it is easy to identify that $p_{i 0, k-1}+p_{i 1, k-1}=1$ for $i=0,1$.

Although the frame length $T F_{k}$ varies with $k$, it holds that $(\alpha+\beta) T F_{k-1} \ll 1$ on the above assumptions. It follows in general that $p_{00, k-1}>p_{01, k-1}$ and $p_{11, k-1}>p_{10, k-1}$. This states an inertia property of the PU activity that $z_{k}$ is more likely to remain the same as $z_{k-1}$ between any two adjacent frames $k-1$ and $k$. In the CR system model, the $\mathrm{SU}$ is assumed to have full knowledge of parameters $\alpha$ and $\beta$ [20,22, 25].

\subsection{Statistics for ED}

We consider the additive white Gaussian noise (AWGN) for the licensed channel. Let $N$ denote the number of signal samples of the SU. For the frame $k$, the $n_{\text {th }}$ baseband signal sample is expressed as

$$
x_{k}(n)= \begin{cases}w_{k}(n), & z_{k}=0 \\ s_{k}(n)+w_{k}(n), & z_{k}=1\end{cases}
$$

where $n=1,2, \ldots, N ; w_{k}(n)$ denotes the independent and identically distributed (i.i.d.) AWGN samples with zero-mean and normalized unit variance; and $s_{k}(n)$ denotes the PU's signal samples with constant transmit power. Hence, the average received signal-tonoise ratio (SNR) per sample at the $\mathrm{SU}$ is expressed as $\gamma=\mathbb{E}\left(\left|s_{k}(n)\right|^{2}\right)$.

As the test statistic of spectrum sensing, the received energy in frame $k$ is given by

$$
U_{k}=\sum_{n=1}^{N}\left|x_{k}(n)\right|^{2}
$$

Then, the test statistic $U_{k}$ follows the chi-square distribution with $N$ degrees of freedom. By the central limit theorem, if the sample length $N$ is large enough $(N \gg 1)$, $U_{k}$ approximately follows the Gaussian distributions

$$
U_{k}= \begin{cases}\mathbb{N}(N, 2 N), & z_{k}=0 \\ \mathbb{N}(N(1+\gamma), 2 N(1+2 \gamma)), & z_{k}=1\end{cases}
$$

where $\mathbb{N}\left(\mu, \sigma^{2}\right)$ is the notation of the Gaussian distribution with mean $\mu$ and variance $\sigma^{2}$.

\subsection{The conventional ED rule}

In most conventional ED schemes, the PU's real states are assumed independent during different frames. Let $d_{k}$ denote the sensing result of the PU's real state $z_{k}$ in frame 
$k$. The decision rule is expressed as a simple comparison between the received energy $U_{K}$ and a threshold $\lambda_{E D, k}$ as follows

$$
d_{k}=\left\{\begin{array}{l}
1, U_{k} \geq \lambda_{E D, k} \\
0, U_{k}<\lambda_{E D, k}
\end{array}\right.
$$

The false alarm probability $P_{f, k}^{E D}$ and the detection probability $P_{d, k}^{E D}$ are obtained as [19]

$$
\begin{gathered}
P_{f, k}^{E D} \triangleq P\left\{d_{k}=1 \mid z_{k}=0\right\}=\mathbb{Q}\left(\frac{\lambda_{E D, k}-N}{\sqrt{2 N}}\right) \\
P_{d, k}^{E D} \triangleq P\left\{d_{k}=1 \mid z_{k}=1\right\}=\mathbb{Q}\left(\frac{\lambda_{E D, k}-N(1+\gamma)}{\sqrt{2 N(1+2 \gamma)}}\right)
\end{gathered}
$$

where $\gamma$ is the average received SNR and

$$
\mathbb{Q}(x)=\frac{1}{\sqrt{2 \pi}} \int_{x}^{\infty} e^{-\frac{t^{2}}{2}} d t
$$

In conventional ED schemes, it is usual to preset a fixed threshold $\lambda_{E D, k}=\lambda_{E D}$ for all $k$. Thus, the probability measures $P_{f, k}^{E D}$ and $P_{d, k}^{E D}$ are independent of $k$. It has been shown that the single fixed threshold scheme yields a high probability of erroneous decision when the received energy $U_{k}$ nears the threshold $\lambda_{E D, k}[11]$.

\section{The IF-DDT scheme}

In this section, we propose an IF-DDT spectrum sensing scheme by exploiting the inertia property of the PU's activity described in Section 2.1. Following the presentation of the decision rule, we formulate an optimization problem to acquire the optimal dynamic double thresholds. Furthermore, we finally transform the optimization problem into an easily solvable problem and derive the optimal dynamic double thresholds with very low complexity.

\subsection{The decision rule using IF-DDT}

To improve the probability of correct decision in the ED schemes, we consider double dynamic thresholds, by which we may elaborate the received energies into three decision ranges in order to exploit the inertia property of the PU's activity. The decision rule using IF-DDT is expressed as follows

$$
d_{k}= \begin{cases}1, & U_{k} \geq \lambda_{1, k} \\ 0, & U_{k}<\lambda_{0, k} \\ d_{k-1}, & \text { otherwise }\end{cases}
$$

where $\lambda_{1, k}$ and $\lambda_{0, k}$ represent the double dynamic thresholds for frame $k$ at the $\mathrm{SU}$, and $\lambda_{0, k} \leqslant \lambda_{1, k}$.

For $k=1$, the two thresholds should be identically initialized as $\lambda_{0,1}=\lambda_{1,1}$. For $k>1$, if the received energy $U_{k}$ lies in the open range $\left(\lambda_{0, k}, \lambda_{1, k}\right)$, the sensing result $d_{k}$ for frame $k$ retains the same as the sensing result $d_{k-1}$ for the preceding frame $k-1$.

It is easy to show that the Markov chain for the PU's activity is reversible according to the steady-state distribution in Eq. (1), the transition matrix in Eq. (2), and hence the detailed balance equation $[24,27]$

$$
P\left\{z_{k-1}=i, z_{k}=j\right\}=P\left\{z_{k}=i, z_{k-1}=j\right\}
$$

Define the reverse transition probability as

$$
p_{i j, k}^{*} \triangleq P\left\{z_{k-1}=j \mid z_{k}=i\right\}
$$

where $i, j \in\{0,1\}$. Then, $p_{i j, k}^{*}=p_{i j, k-1}$ is derived.

According to the decision rule using IF-DDT in Eq. (9), the probabilities $P_{f, k}$ and $P_{d, k}$ are obtained as follows (see Appendix 1)

$$
\begin{aligned}
P_{f, k}= & \mathbb{Q}\left(\frac{\lambda_{0, k}-N}{\sqrt{2 N}}\right) P_{0, k}+\mathbb{Q}\left(\frac{\lambda_{1, k}-N}{\sqrt{2 N}}\right)\left(1-P_{0, k}\right) \\
P_{d, k}= & \mathbb{Q}\left(\frac{\lambda_{0, k}-N(1+\gamma)}{\sqrt{2 N(1+2 \gamma)}}\right) P_{1, k} \\
& +\mathbb{Q}\left(\frac{\lambda_{1, k}-N(1+\gamma)}{\sqrt{2 N(1+2 \gamma)}}\right)\left(1-P_{1, k}\right)
\end{aligned}
$$

where

$$
\begin{aligned}
& P_{0, k} \triangleq P\left\{d_{k-1}=1 \mid z_{k}=0\right\}=P_{f, k-1} p_{00, k}^{*}+P_{d, k-1} p_{01, k}^{*} \\
& P_{1, k} \triangleq P\left\{d_{k-1}=1 \mid z_{k}=1\right\}=P_{f, k-1} p_{10, k}^{*}+P_{d, k-1} p_{11, k}^{*}
\end{aligned}
$$

For ease of understanding the IF-DDT scheme, Fig. 2 shows the definitions for multiple events and associated probabilistic measures on the reversible Markov chain.

\subsection{Derivation of thresholds}

To derive the double thresholds, there exists two hypothesis testing criterions: the Neyman-Pearson (NP) test [8] and the Bayesian test [28]. We consider only the NP test criterion, which aims at maximizing $P_{d, k}$ with the constraint $P_{f, k} \leq P_{f \text {,target }}$, or alternatively minimizing $P_{f, k}$ with the constraint $P_{d, k} \geq P_{d, \text { target }}$, where $P_{f \text {,target }}$ and $P_{d \text {,target }}$ represent the tolerable maximum false alarm probability and minimum detection probability, respectively.

For any sensing frame $k>1$, the probabilities $P_{0, k}$ and $P_{1, k}$ are considered constant since $p_{i j, k}^{*}$ are determined by Eq. (2), and $P_{f, k-1}$ and $P_{d, k-1}$ are obtained at frame $k-1$. Thus, by Eqs. (11) and (12), it is clear that $P_{f, k}$ and $P_{d, k}$ are strictly decreasing with $\lambda_{0, k}$ and $\lambda_{1, k}$, respectively. According to the NP test criterion, the search for 


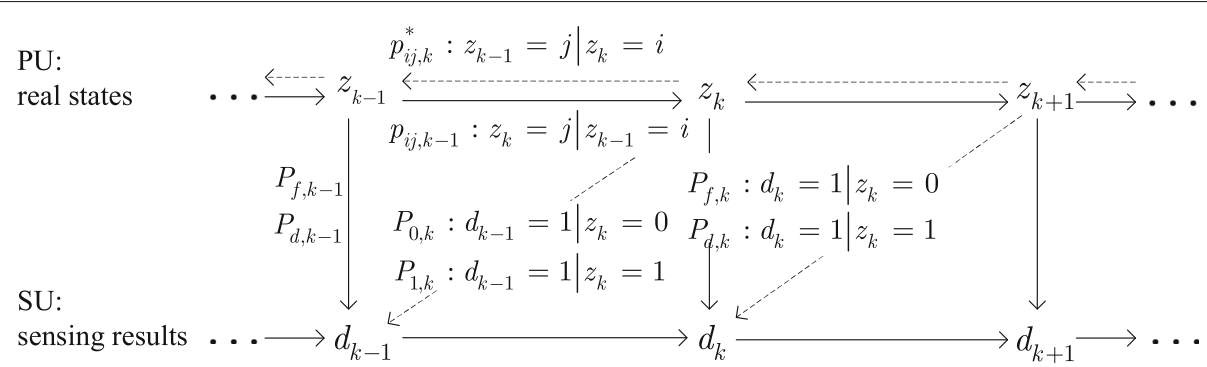

Fig. 2 Probabilities and transitions associated with multiple events in the reversible Markov chain model

the optimal thresholds $\lambda_{0, k}^{*}$ and $\lambda_{1, k}^{*}$ with respect to each frame $k$ is formulated as the optimization problem below

$$
\begin{array}{ll}
\max _{0, k}, \lambda_{1, k} & P_{d, k}\left(\lambda_{0, k}, \lambda_{1, k}\right) \\
\text { s.t. } & \lambda_{1, k} \geq \lambda_{0, k} \geq 0 \\
& P_{f, k}\left(\lambda_{0, k}, \lambda_{1, k}\right) \leq P_{f, \text { target }}
\end{array}
$$

where $P_{d, k}$ and $P_{f, k}$ are written as bivariate functions of thresholds.

Theorem $1 P_{d, k}$ reaches the maximum value $P_{d, k}^{*}$ only when $P_{f, k}=P_{f, \text { target }}$, i.e., the optimization problem in Eq. (15) can be rewritten as (proved in Appendix 2)

$$
\begin{array}{ll}
\max _{0, k}, \lambda_{1, k} & P_{d, k}\left(\lambda_{0, k}, \lambda_{1, k}\right) \\
\text { s.t. } & \lambda_{1, k} \geq \lambda_{0, k} \geq 0 \\
& P_{f, k}\left(\lambda_{0, k}, \lambda_{1, k}\right)=P_{f, \text { target }}
\end{array}
$$

Based on the second constraint in Eq. (16), we define the Lagrange function

$$
\begin{aligned}
f\left(\lambda_{0, k}, \lambda_{1, k}, c\right)= & P_{d, k}\left(\lambda_{0, k}, \lambda_{1, k}\right) \\
& +c\left(P_{f, \text { target }}-P_{f, k}\left(\lambda_{0, k}, \lambda_{1, k}\right)\right)
\end{aligned}
$$

where $c$ is the Lagrange multiplier.

Taking the first-order partial derivative of Eq. (17) with respect to $\lambda_{0, k}$ and $\lambda_{1, k}$, respectively

$$
\frac{\partial f\left(\lambda_{0, k}, \lambda_{1, k}, c\right)}{\partial \lambda_{0, k}}=0, \quad \frac{\partial f\left(\lambda_{0, k}, \lambda_{1, k}, c\right)}{\partial \lambda_{1, k}}=0
$$

then

$$
\left\{\begin{array}{l}
\frac{1}{\sqrt{1+2 \gamma}} e^{\frac{\left(\lambda_{0, k}-N\right)^{2}}{4 N}-\frac{\left[\lambda_{0, k}-N(1+\gamma)\right]^{2}}{4 N(1+2 \gamma)}}=c \frac{P_{0, k}}{P_{1, k}} \\
\frac{1}{\sqrt{1+2 \gamma}} e^{\frac{\left(\lambda_{1, k}-N\right)^{2}}{4 N}-\frac{\left[\lambda_{1, k}-N(1+\gamma)\right]^{2}}{4 N(1+2 \gamma)}}=c \frac{1-P_{0, k}}{1-P_{1, k}}
\end{array}\right.
$$

Eliminating $c$ in Eq. (19) by simple manipulations, we get

$$
\left(\lambda_{1, k}-a\right)^{2}-\left(\lambda_{0, k}-a\right)^{2}=b
$$

where

$$
a=\frac{N}{2}, \quad b=\frac{2 N(1+2 \gamma)}{\gamma} \ln \frac{P_{1, k}\left(1-P_{0, k}\right)}{P_{0, k}\left(1-P_{1, k}\right)}
$$

Then, recalling the second constraint in Eq. (16), we obtain

$$
\mathbb{Q}\left(\frac{\lambda_{0, k}-N}{\sqrt{2 N}}\right) P_{0, k}+\mathbb{Q}\left(\frac{\lambda_{1, k}-N}{\sqrt{2 N}}\right)\left(1-P_{0, k}\right)=P_{f, \text { target }}
$$

So we convert the optimization problem in Eq. (16) into a nonlinear programming problem consisting of Eqs. (20) and (22).

Clearly, the existence of the solution to the optimization problem depends upon the system parameters, including the sample length $N$, the average $S N R$ value $\gamma$, the frame lengths $T F_{k}$, the expectation of ON and OFF state period $\bar{T}_{1}, \bar{T}_{0}$, and the target false alarm probability $P_{f \text {,target }}$ On the other hand, the solution to the nonlinear programming problem cannot be expressed in a closed form. Therefore, in order to facilitate a heuristic approach for the solution, we derive the range for the above nonlinear programming problem firstly.

Notice that the function $\mathbb{Q}(x)$ is a strictly decreasing function. Under the initial constraint that $\lambda_{1, k} \geq \lambda_{0, k} \geq 0$ and the basic probability property that $0<P_{0, k}, 1-P_{0, k}<$ 1 , we can derive a necessary condition on the threshold $\lambda_{0, k}$ below

$\min \left[\frac{P_{f, \text { target }}}{P_{0, k}}, \mathbb{Q}\left(-\sqrt{\frac{N}{2}}\right)\right] \geq \mathbb{Q}\left(\frac{\lambda_{0, k}-N}{\sqrt{2 N}}\right) \geq P_{f, \text { target }}$

Thus, the range of $\lambda_{0, k}$ is $\left[\lambda_{0, k}^{*}, \lambda^{*}\right]$,it is given by

$$
\lambda_{0, k}^{*} \triangleq \sqrt{2 N} \mathbb{Q}^{-1}\left\{\min \left[\frac{P_{f, \text { target }}}{P_{0, k}}, \mathbb{Q}\left(-\sqrt{\frac{N}{2}}\right)\right]\right\}+N
$$

$$
\lambda^{*} \triangleq \sqrt{2 N} \mathbb{Q}^{-1}\left(P_{f, \text { target }}\right)+N
$$


With incorporating the range of $\lambda_{0, k}$, we obtain a necessary condition on the threshold $\lambda_{1, k}$ as follows

$$
P_{f, \text { target }} \geq \mathbb{Q}\left(\frac{\lambda_{1, k}-N}{\sqrt{2 N}}\right) \geq \frac{P_{f, \text { target }}-\mathbb{Q}\left(\frac{\lambda_{0, k}^{*}-N}{\sqrt{2 N}}\right) P_{0, k}}{1-P_{0, k}}
$$

and the range $\left[\lambda^{*}, \lambda_{1, k}^{*}\right]$ of $\lambda_{1, k}$ is given by

$$
\lambda_{1, k}^{*} \triangleq \sqrt{2 N} \mathbb{Q}^{-1}\left(\frac{P_{f, \text { target }}-\mathbb{Q}\left(\frac{\lambda_{0, k}^{*}-N}{\sqrt{2 N}}\right) P_{0, k}}{1-P_{0, k}}\right)+N
$$

In practice, it is easy to show that $\lambda^{*}>a$ since $P_{f \text {,target }}<0.5$ and $\mathbb{Q}^{-1}\left(P_{f \text {,target }}\right)>0$. By analysing Eq. (20) and Eq. (24), we can obtain the relationship of $\lambda_{1, k}^{*}$, $\lambda_{0, k}^{*}$, and $a$ as follows:

$$
\begin{cases}\lambda_{0, k}^{*}=0, \lambda_{1, k}^{*}<+\infty & \frac{P_{f, \text { target }}}{P_{0, k}} \geq \mathbb{Q}\left(-\sqrt{\frac{N}{2}}\right) \\ 0<\lambda_{0, k}^{*} \leq a, \lambda_{1, k}^{*} \rightarrow+\infty & \mathbb{Q}\left(-\frac{1}{2} \sqrt{\frac{N}{2}}\right) \leq \frac{P_{f, \text { target }}}{P_{0, k}}<\mathbb{Q}\left(-\sqrt{\frac{N}{2}}\right) \\ \lambda_{0, k}^{*}>a, \lambda_{1, k}^{*} \rightarrow+\infty & \frac{P_{f, \text { target }}}{P_{0, k}}<\mathbb{Q}\left(-\frac{1}{2} \sqrt{\frac{N}{2}}\right)\end{cases}
$$

Combined with the range of $\lambda_{0, k}$ and $\lambda_{1, k}$ in three cases in Eqs.(28), (20), and (21) can be further demonstrated in the two-dimension Cartesian coordinate system as showed in Fig. 3. When $\frac{P_{f, \text { target }}}{P_{0, k}} \geq \mathbb{Q}\left(-\sqrt{\frac{N}{2}}\right)$, Eqs. (20) and (21) are demonstrated in Fig. 3a; when $\mathbb{Q}\left(-\frac{1}{2} \sqrt{\frac{N}{2}}\right) \leq$ $\frac{P_{f, \text { target }}}{P_{0, k}}<\mathbb{Q}\left(-\sqrt{\frac{N}{2}}\right)$, Eqs. (20) and (21) are demonstrated in Fig. 3b; and when $\frac{P_{f, \text { target }}}{P_{0, k}}<\mathbb{Q}\left(-\frac{1}{2} \sqrt{\frac{N}{2}}\right)$, Eqs. (20) and (21) are demonstrated in Fig. 3c.

\subsection{Solution analysis}

Based on Eqs. (20) and (21), $\lambda_{1, k}$ can respectively be seen as the functions of $\lambda_{0, k}$ within the range obtained above. First, $g_{1}\left(\lambda_{0, k}\right)$ and $g_{2}\left(\lambda_{0, k}\right)$ are defined respectively as

$$
g_{1}\left(\lambda_{0, k}\right) \triangleq \sqrt{2 N} \mathbb{Q}^{-1}\left(\frac{P_{f, \text { target }}-\mathbb{Q}\left(\frac{\lambda_{0, k}-N}{\sqrt{2 N}}\right) P_{0, k}}{1-P_{0, k}}\right)+N
$$

$$
g_{2}\left(\lambda_{0, k}\right) \triangleq \sqrt{\left(\lambda_{0, k}-a\right)^{2}+b}+a
$$

And $g\left(\lambda_{0, k}\right)$ is further defined as

$$
g\left(\lambda_{0, k}\right) \triangleq g_{1}\left(\lambda_{0, k}\right)-g_{2}\left(\lambda_{0, k}\right)
$$

Obviously, the zero point of $g\left(\lambda_{0, k}\right)$ is the solution of $\lambda_{0, k}$ to the nonlinear programming problem in Eqs. (20) and (21) meanwhile.

The first-order differential function of $g\left(\lambda_{0, k}\right)$ is then deduced as

$$
\begin{aligned}
g^{\prime}\left(\lambda_{0, k}\right) & \triangleq g_{1}^{\prime}\left(\lambda_{0, k}\right)-g_{2}^{\prime}\left(\lambda_{0, k}\right) \\
& =-\frac{P_{0, k}}{1-P_{0, k}} e^{\frac{1}{4 N}\left[\left(\lambda_{1, k}-N\right)^{2}-\left(N-\lambda_{0, k}\right)^{2}\right]}-\frac{\lambda_{0, k}-a}{\lambda_{1, k}-a}
\end{aligned}
$$

where $\lambda_{1, k}=\sqrt{2 N} \mathbb{Q}^{-1}\left(\frac{P_{f \text { target }}-\mathbb{Q}\left(\frac{\lambda_{0, k}-N}{\sqrt{2 N}}\right) P_{0, k}}{1-P_{0, k}}\right)+N$.

Together with Eq. (28), the negativity of $g^{\prime}\left(\lambda_{0, k}\right)$ in the range of three cases in Eq. (28) is proved as follows.

(1) $\frac{P_{f, \text { target }}}{P_{0, k}}<\mathbb{Q}\left(-\frac{1}{2} \sqrt{\frac{N}{2}}\right)$. It can be easily obtained that $\frac{\lambda_{0, k}-a}{\lambda_{1, k}-a}>0$, and thus, we can get $g^{\prime}\left(\lambda_{0, k}\right)<0$.

(2) $\frac{P_{f, \text { target }}}{P_{0, k}} \geq \mathbb{Q}\left(-\frac{1}{2} \sqrt{\frac{N}{2}}\right)$.

(i) $\lambda_{0, k} \in\left(a, \lambda^{*}\right)$. Similar to (1), it can be obtained that $g^{\prime}\left(\lambda_{0, k}\right)<0$.
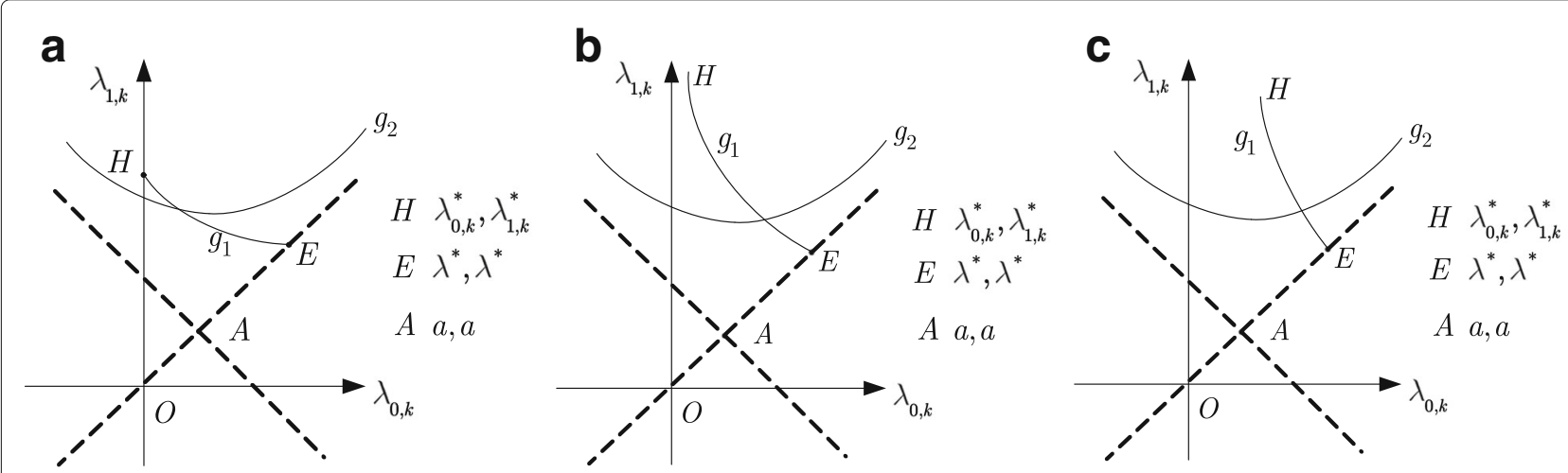

Fig. 3 a-c Analysis of the solution to the nonlinear programming problem 
(ii) $\lambda_{0, k} \in\left(\lambda_{0, k}^{*}, a\right)$, Eq. (20) can be transformed into

$$
\begin{aligned}
& {\left[1-\frac{1}{2} \operatorname{erfc}\left(\frac{N-\lambda_{0, k}}{2 \sqrt{N}}\right)\right] P_{0, k}} \\
& \quad+\frac{1}{2} \operatorname{erfc}\left(\frac{\lambda_{1, k}-N}{2 \sqrt{N}}\right)\left(1-P_{0, k}\right)=P_{f, \text { target }}
\end{aligned}
$$

$$
\text { where } \operatorname{erf} c(x)=2 \mathbb{Q}(\sqrt{2} x) \text {. }
$$

With $\lambda_{0, k}<a$, it can be deduced that

$$
\frac{N-\lambda_{0, k}}{2 \sqrt{N}}>\frac{N-a}{2 \sqrt{N}}=\frac{\sqrt{N}}{4}
$$

and

$$
\begin{aligned}
\frac{\lambda_{1, k}-N}{2 \sqrt{N}} & =\frac{1}{\sqrt{2}} \mathbb{Q}^{-1}\left(\frac{P_{f, \text { target }}-\mathbb{Q}\left(\frac{\lambda_{0, k}-N}{\sqrt{2 N}}\right) P_{0, k}}{1-P_{0, k}}\right) \\
& >\frac{1}{\sqrt{2}} \mathbb{Q}^{-1}\left(\frac{P_{f, \text { target }}-\mathbb{Q}\left(-\frac{\sqrt{N}}{2 \sqrt{2}}\right) P_{0, k}}{1-P_{0, k}}\right) \\
& >\frac{1}{\sqrt{2}} \mathbb{Q}^{-1}\left(\frac{P_{0, k}-\mathbb{Q}\left(-\frac{\sqrt{N}}{2 \sqrt{2}}\right) P_{0, k}}{1-P_{0, k}}\right) \\
& >\frac{1}{\sqrt{2}} \mathbb{Q}^{-1}\left(1-\mathbb{Q}\left(-\frac{\sqrt{N}}{2 \sqrt{2}}\right)\right) \\
& =\frac{\sqrt{N}}{4}
\end{aligned}
$$

Notice that in Eq. (35), $P_{0, k}>P_{f \text {,target }}$ since $P_{d, k-1}>$ $P_{f \text {,target }}$ must hold to ensure a valid detection and $P_{f, k-1}=P_{f \text {,target }}$ in Eq. (13). With $\gamma \ll 1$ and $N \gg 1$, the approximation $\operatorname{er} f(x) \approx \frac{1}{\sqrt{\pi} x} e^{-x^{2}}$ can be taken in Eq. (33), and thus, we can get

$$
\begin{aligned}
& \frac{P_{0, k}}{1-P_{0, k}} e^{\frac{1}{4 N}\left[\left(\lambda_{1, k}-N\right)^{2}-\left(N-\lambda_{0, k}\right)^{2}\right]} \\
& \quad=\frac{N-\lambda_{0, k}}{\lambda_{1, k}-N}+\frac{P_{0, k}-P_{f, \text { target }}}{1-P_{0, k}} \sqrt{\frac{\pi}{N}}\left(N-\lambda_{0, k}\right) e^{\frac{1}{4 N}\left(\lambda_{1, k}-N\right)^{2}}
\end{aligned}
$$

By taking Eq. (36) into Eq. (32), it can be proved that $g^{\prime}\left(\lambda_{0, k}\right)<0$ when $\lambda_{0, k} \in\left(\lambda_{0, k}^{*}, a\right)$; the nonlinear programming problem is then transformed into a problem of searching for the zero point of a strictly decreasing function, which can be easily solved by the dichotomy or Newton's method. It should be noticed that no zero point exists when $g\left(\lambda_{0, k}^{*}\right)<0$ since $g\left(\lambda^{*}\right)<0$.

\section{Simulation results}

In this section, numerical results are presented to evaluate the effectiveness of the IF-DDT sensing scheme. The proposed algorithm is tested under various average activity periods and traffic loads of the PU.

\subsection{Simulation setup}

Firstly, the thresholds of the IF-DDT scheme are simulated. Secondly, the actual false alarm of the new algorithm is provided to examine the correctness of the thresholds. Thirdly, the performance of the IF-DDT algorithm is quantified in different average PU active periods and PU traffic loads, respectively. Finally, the total error probability of the IF-DDT scheme is provided to verify the detection performance.

In the simulations, the sample length $N$ is set to be 1000 , the sensing frame length $T F_{k}$ is set to be $10 \mathrm{~ms}$ according to the IEEE 802.22 standard, and a Markov chain with $10^{6}$ frames is randomly generated to model the PU state varying in each simulation. The detection performance of the proposed IF-DDT scheme is compared with that of the ED scheme and the double-threshold (DT) energy scheme in [11].

\subsection{Numerical results}

Figure 4 verifies the double thresholds of the IF-DDT algorithm changing with the frame index $k$. Figure $4 a$, b is plotted at low and high SNR, respectively. In both of the subfigures, $\bar{T}_{0}=\bar{T}_{1}=200 \mathrm{~ms}$ is set. In addition, the gray areas in the subfigures denote that the real state of the PU is ON; otherwise, it is OFF. When the received energy locates between the double thresholds and correctly detected under the IF-DDT scheme, the corresponding point is marked with a circle.

From Fig. 4a, b, we can see that the double thresholds quickly converge to stable values and actually keep invariant. It means that the calculation for the thresholds of the subsequent sensing frames can be saved as long as the system parameters remain unchanged. Furthermore, the marked points show that in lots of frames where the received energy cannot give a reliable local decision, the conventional ED algorithm seem powerless but our algorithm tends to make the right judgment. It is also indicated that the gap between the double thresholds is smaller when the SNR is high.

Figure 5 tests the actual false alarm probability of the ED scheme, IF-DDT scheme, and DT scheme versus SNR when $P_{f \text {,target }}=0.01,0.05,0.1$, respectively. Other simulation settings are $\bar{T}_{0}=\bar{T}_{1}=200 \mathrm{~ms}$. As shown in the figure, the actual false alarm probability of the DT scheme is slightly higher than the target false alarm probability, but the actual false alarm probability of the IF-DDT 


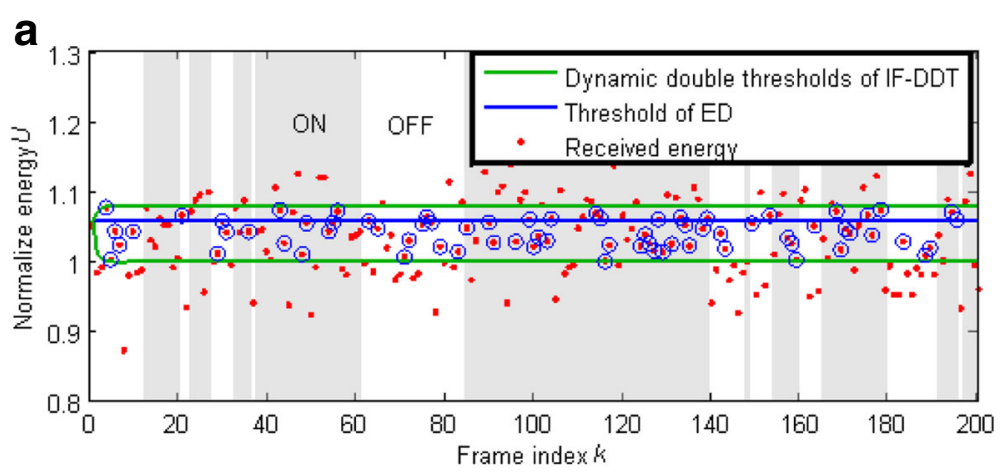

b

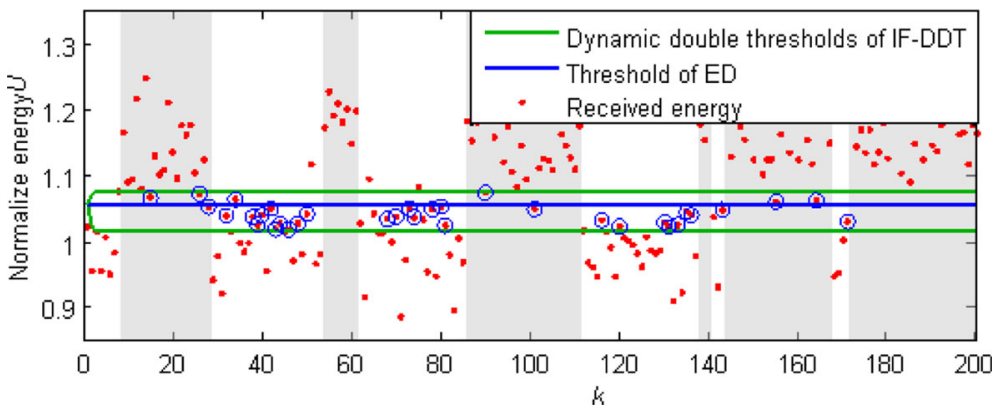

Fig. 4 Thresholds of the IF-DDT sensing scheme. a Thresholds for $\bar{T}_{0}=\bar{T}_{1}=200 \mathrm{~ms}$ and SNR $=-14 \mathrm{~dB}$. Thresholds of the IF-DDT sensing scheme. $\mathbf{b}$ Thresholds for $\bar{T}_{0}=\bar{T}_{1}=200 \mathrm{~ms}$ and SNR $=-8 \mathrm{~dB}$

scheme and ED scheme is always equal to the target false alarm probability. In other words, the dynamic double thresholds derived from Eq. (15) ensure that the constraint on false alarm probability is met.

Figure 6 shows the detection probability versus SNR with different $\mathrm{PU}$ active periods. The average PU active parameters are set to be $\bar{T}_{0}=\bar{T}_{1}=100,200,500 \mathrm{~ms}$, respectively. As shown in Fig. 6 , the IF-DDT algorithm shows superior performance to the conventional ED and

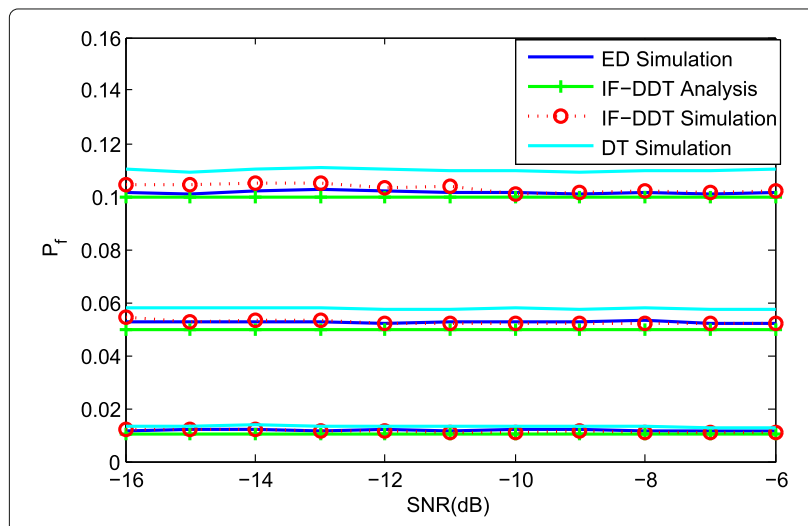

Fig. 5 False alarm probability testing for $\bar{T}_{0}=\bar{T}_{1}=200 \mathrm{~ms}$
DT scheme even when the average period of the PU is short. For example, at the SNR of $-10 \mathrm{~dB}$, the detection probability of the IF-DDT algorithm reaches 0.92 when $\bar{T}_{0}=\bar{T}_{1}=100 \mathrm{~ms}$, comparing with $P_{d}^{E D}=0.81$ for the ED scheme and $P_{d}^{D T}=0.84$ for the DT scheme.

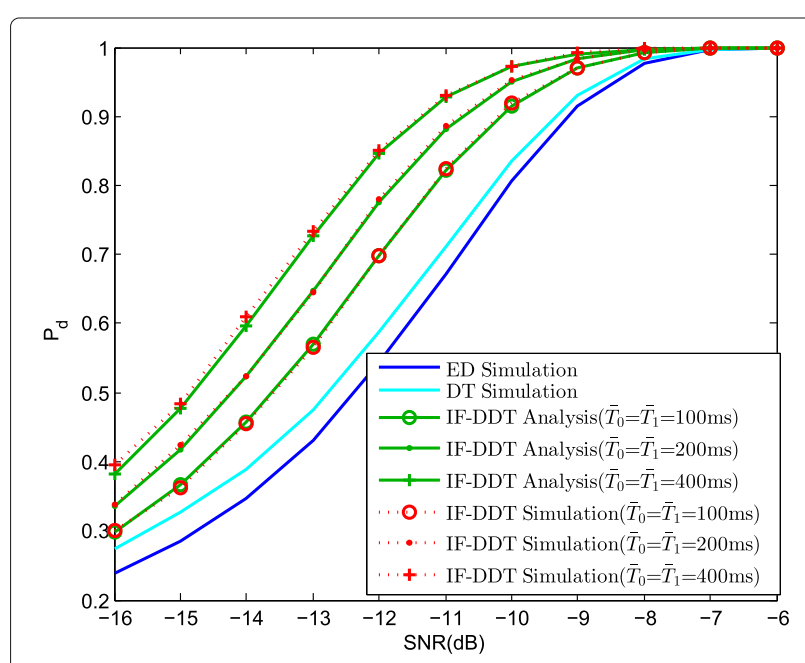

Fig. $6 P_{d}$ versus $S N R$ for $P_{f \text {,target }}=0.1$ and $\eta_{\mathrm{PU}}=0.5$ 
This improvement benefits from the dynamic doublethreshold strategy, with which the detector tends to make the correct judgment when the received energy is not reliable enough. What is more, when the PU actives with longer period and thus becomes more inertial, the IFDDT algorithm gains higher detection probability which depicts that the PU active period has much impact on the performance of the new sensing scheme. In addition, it is clearly shown that the simulation results well coincide with the theoretical analysis in all cases.

Figure 7 tests the performance of the IF-DDT scheme when the PU's traffic load varies. And as expected, the new scheme gains obvious performance improvement compared with the convention ED and DT schemes. It is also verified that when the PU's average activity period is fixed, the detection probability of the proposed algorithm does not vary much with the PU's traffic load. For example, at the SNR of $-12 \mathrm{~dB}$, the detection probability is 0.78 , which is slightly higher by 0.03 and 0.06 , respectively, than that in the other cases. The difference is even slighter in higher SNR region.

It is important to investigate the detector's capability on timely and correctly identifying the spectrum hole when the spectrum is not occupied by the PU and vacating the spectrum when the PU starts to occupy the authorized spectrum. Therefore, to compare the performance in terms of both the detection probability and the false alarm probability, we define an erroneous decision probability $P_{e}$ as follows:

$$
P_{e}=P_{\mathrm{OFF}} P_{f}+P_{\mathrm{ON}}\left(1-P_{d}\right)
$$

Figure 8 shows $P_{e}$ among IF-DDT scheme, ED scheme, and DT scheme versus SNR. The simulation environment is the same as that in Fig. 6 . The figure shows that IFDDT scheme provide the lowest $P_{e}$ among these schemes. In addition, the $P_{e}$ of the IF-DDT scheme decreases as the average period of the PU becomes longer. This is due to the fact that when the average period increases, the PU's state between contiguous frames tends to be more inertial, and then, the decision rule in Eq. (9) is more reliable; therefore, the decision based on the energy falling between the two thresholds is more reasonable. Furthermore, the performance of ED and DT schemes are much worse than those of the IF-DDT scheme.

Figures 6, 7, and 8 reveal:

1. IF-DDT sensing scheme outperforms the conventional ED scheme and DT scheme

2. The proposed method is more robust to the PU's activity period and traffic load.

\section{Conclusions}

In this paper, the PU's activity model and the SU's sensing mechanism are utilized and an inter-frame-based dynamic double-threshold spectrum sensing scheme, which exploits the relationship of two adjacent sensing frames, is proposed to enhance energy detection performance. When the received energy cannot give a reliable local decision to distinguish the ON and OFF states of the $\mathrm{PU}$, the detector retains the previous sensing result. The detection probability and false alarm probability of the proposed sensing scheme are analyzed, and the optimal

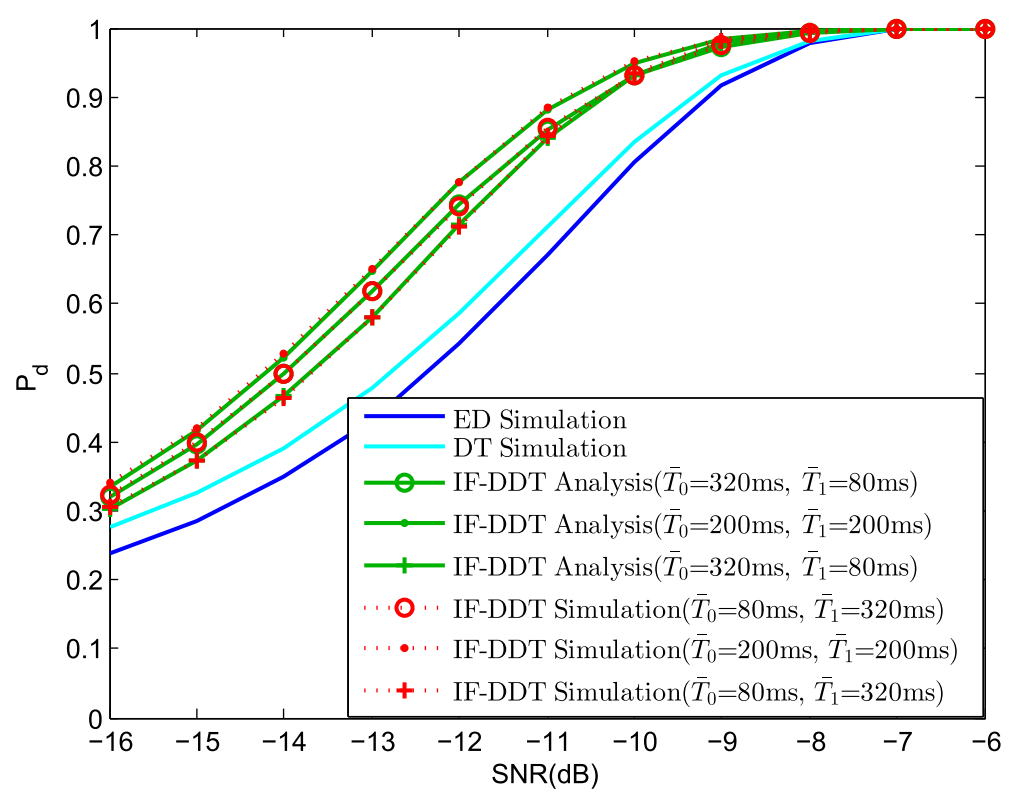

Fig. $7 P_{d}$ versus $S N R$ for $P_{f \text {,target }}=0.1$ and $T_{\mathrm{PU}}=400 \mathrm{~ms}$ 


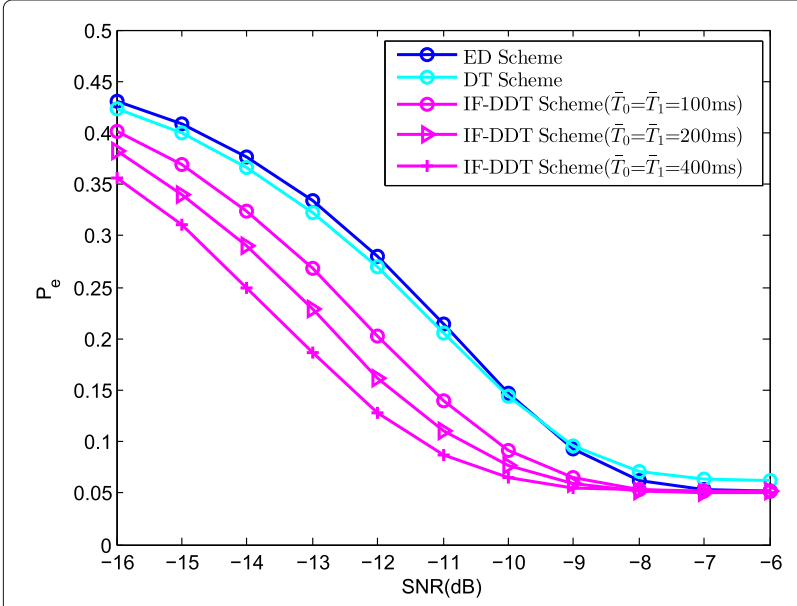

Fig. $8 P_{e}$ versus $S N R$ for $P_{f \text {,target }}=0.1$ and $\eta_{\mathrm{PU}}=0.5$

double thresholds are derived with very low complexity according to the Neyman-Pearson (NP) test criterion. This sensing scheme is simple but shown to outperform the ED algorithm from both theoretical analysis and simulation results.

In this paper, our algorithm is based on energy detection. However, it can be extended to other traditional spectrum sensing method, such as coherent detection and feature detection, where the PU's activity model and the SU's sensing mechanism have not been exploited. The same way can be followed to obtain the corresponding dynamic double thresholds and the detection probability together with the false alarm probability.

\section{Appendix 1: Derivation of Eqs. (12) and (13)}

According to the definition for $P_{f, k}$ and the decision rule given by Eq. (9),

$$
\begin{aligned}
P_{f, k} \triangleq & P\left\{d_{k}=1 \mid z_{k}=0\right\} \\
= & P\left\{U_{k} \geq \lambda_{1, k} \mid z_{k}=0\right\} \\
& +P\left\{\lambda_{0, k}<U_{k}<\lambda_{1, k}, d_{k-1}=1 \mid z_{k}=0\right\} \\
= & P\left\{U_{k} \geq \lambda_{1, k} \mid z_{k}=0\right\} \\
& +P\left\{\lambda_{0, k}<U_{k}<\lambda_{1, k} \mid z_{k}=0\right\} P\left\{d_{k-1}=1 \mid z_{k}=0\right\} \\
= & \mathbb{Q}\left(\frac{\lambda_{1, k}-N}{\sqrt{2 N}}\right)+\left[\mathbb{Q}\left(\frac{\lambda_{0, k}-N}{\sqrt{2 N}}\right)-\mathbb{Q}\left(\frac{\lambda_{1, k}-N}{\sqrt{2 N}}\right)\right] P_{0, k}
\end{aligned}
$$

where

$$
\begin{aligned}
P_{0, k} \triangleq & P\left\{d_{k-1}=1 \mid z_{k}=0\right\} \\
= & P\left\{d_{k-1}=1, z_{k-1}=0 \mid z_{k}=0\right\} \\
& +P\left\{d_{k-1}=1, z_{k-1}=1 \mid z_{k}=0\right\} \\
= & P\left\{d_{k-1}=1 \mid z_{k-1}=0\right\} P\left\{z_{k-1}=0 \mid z_{k}=0\right\} \\
& +P\left\{d_{k-1}=1 \mid z_{k-1}=1\right\} P\left\{z_{k-1}=1 \mid z_{k}=0\right\} \\
= & P_{f, k-1} p_{00, k}^{*}+P_{d, k-1} p_{01, k}^{*}
\end{aligned}
$$

Similarly, the detection probability $P_{d, k}$ can be derived as

$$
\begin{aligned}
P_{d, k} \triangleq & P\left\{d_{k}=1 \mid z_{k}=1\right\} \\
= & P\left\{U_{k} \geq \lambda_{1, k} \mid z_{k}=1\right\} \\
& +P\left\{\lambda_{0, k}<U_{k}<\lambda_{1, k}, d_{k-1}=1 \mid z_{k}=1\right\} \\
= & P\left\{U_{k} \geq \lambda_{1, k} \mid z_{k}=1\right\} \\
& +P\left\{\lambda_{0, k}<U_{k}<\lambda_{1, k} \mid z_{k}=1\right\} P\left\{d_{k-1}=1 \mid z_{k}=1\right\} \\
= & \mathbb{Q}\left(\frac{\lambda_{1, k}-N(1+\gamma)}{\sqrt{2 N(1+2 \gamma)}}\right) \\
& +\left[\mathbb{Q}\left(\frac{\lambda_{0, k}-N(1+\gamma)}{\sqrt{2 N(1+2 \gamma)}}\right)-\mathbb{Q}\left(\frac{\lambda_{1, k}-N(1+\gamma)}{\sqrt{2 N(1+2 \gamma)}}\right)\right] P_{1, k}
\end{aligned}
$$

and

$$
P_{1, k} \triangleq P\left\{d_{k-1}=1 \mid z_{k}=1\right\}=P_{f, k-1} p_{10, k}^{*}+P_{d, k-1} p_{11, k}^{*}
$$

\section{Appendix 2: Proof of Theorem 1}

Consider first $k>1$. In Eqs. (10) and (11), $P_{0, k}$ and $P_{1, k}$ can be viewed as constants in the open range $(0,1)$. Thus, the probabilities $P_{f, k}$ and $P_{d, k}$ are both strictly decreasing functions of $\lambda_{0, k}$ and $\lambda_{1, k}$, respectively.

Assume that $\left(\lambda_{0, k}^{\prime}, \lambda_{1, k}^{\prime}\right)$ is the optimal solution to Eq. (16) such that $\lambda_{0, k}^{\prime}<\lambda_{1, k}^{\prime}, P_{f, k}\left(\lambda_{0, k}^{\prime}, \lambda_{1, k}^{\prime}\right)<P_{f \text {,target }}$, and $P_{d, k}\left(\lambda_{0, k}^{\prime}, \lambda_{1, k}^{\prime}\right)=P_{d, k}^{*}$, where $P_{d, k}^{*}$ is the maximum value for $P_{d, k}$. Then, there must exist a smaller value $\lambda_{0, k}^{*}<\lambda_{0, k}^{\prime}$ such that

$$
\begin{array}{r}
P_{f, k}\left(\lambda_{0, k}^{\prime}, \lambda_{1, k}^{\prime}\right)<P_{f, k}\left(\lambda_{0, k}^{*}, \lambda_{1, k}^{\prime}\right)=P_{f, \text { target }} \\
P_{d, k}\left(\lambda_{0, k}^{\prime}, \lambda_{1, k}^{\prime}\right)=P_{d, k}^{*}<P_{d, k}\left(\lambda_{0, k}^{*}, \lambda_{1, k}^{\prime}\right)
\end{array}
$$

which contradicts the assumption of the maximum value $P_{d, k}^{*}$

On the other hand, if $\lambda_{0, k}^{\prime}<\lambda_{1, k}^{\prime}$, we may also reduce $\lambda_{1, k}^{\prime}$ to $\lambda_{1, k}^{*} \in\left[\lambda_{0, k}^{\prime}, \lambda_{1, k}^{\prime}\right)$ with $\lambda_{0, k}^{\prime}$ given, such that $P_{f, k}\left(\lambda_{0, k}^{\prime}, \lambda_{1, k}^{*}\right)=P_{f, \text { target }}$, followed by $P_{d, k}\left(\lambda_{1, k}^{*}, \lambda_{0, k}^{\prime}\right)>$ $P_{d, k}^{*}$

In the special case $k=1$, the probabilities $P_{f, 1}$ and $P_{d, 1}$ become strictly decreasing functions of $\lambda_{1}$ as described underneath Eq. (9). Similarly, it can be proved that $P_{d, 1}$ reaches $P_{d, 1}^{*}$ only when $P_{f, 1}=P_{f \text {,target }}$ using a proper threshold $\lambda_{1}^{*}$.

Therefore, it is concluded that $P_{d, k}$ reaches the maximum value $P_{d, k}^{*}$ only when $P_{f, k}=P_{f \text {,target }}$ with pair thresholds $\left(\lambda_{0, k}^{*}, \lambda_{1, k}^{*}\right)$ for all $k>1$. 


\section{Appendix 3: Proof of Eq. (23)}

The proof is composed of two steps:

Firstly, $\frac{P_{f, \text { target }}}{P_{0, k}} \geq \mathbb{Q}\left(\frac{\lambda_{0, k}-N}{\sqrt{2 N}}\right)$ can be derived from

Eq. (20). From $\frac{\lambda_{0, k}-N}{\sqrt{2 N}}=-\sqrt{\frac{N}{2}}+\frac{\lambda_{0, k}}{\sqrt{2 N}} \geq-\sqrt{\frac{N}{2}}$ and the monotonic decreasing property of $\mathbb{Q}$ function, we obtain $\mathbb{Q}\left(-\sqrt{\frac{N}{2}}\right) \geq \mathbb{Q}\left(\frac{\lambda_{0, k}-N}{\sqrt{2 N}}\right)$.

Therefore $\min \left[\frac{P_{f, \text { target }}}{P_{0, k}}, \mathbb{Q}\left(-\sqrt{\frac{N}{2}}\right)\right] \geq \mathbb{Q}\left(\frac{\lambda_{0, k}-N}{\sqrt{2 N}}\right)$.

Secondly, from $\lambda_{0, k} \leq \lambda_{1, k}$, we have $\frac{\lambda_{0, k}-N}{\sqrt{2 N}} \leq \frac{\lambda_{1, k}-N}{\sqrt{2 N}}$, then $\mathbb{Q}\left(\frac{\lambda_{0, k}-N}{\sqrt{2 N}}\right) \geq \mathbb{Q}\left(\frac{\lambda_{0, k}-N}{\sqrt{2 N}}\right)$.

On the one hand, $\mathbb{Q}\left(\frac{\lambda_{0, k}-N}{\sqrt{2 N}}\right) P_{0, k}+\mathbb{Q}\left(\frac{\lambda_{0, k}-N}{\sqrt{2 N}}\right)(1-$ $\left.P_{0, k}\right)=\mathbb{Q}\left(\frac{\lambda_{0, k}-N}{\sqrt{2 N}}\right)$. On the other hand, $\mathbb{Q}\left(\frac{\lambda_{0, k}-N}{\sqrt{2 N}}\right) P_{0, k}+$ $\mathbb{Q}\left(\frac{\lambda_{0, k}-N}{\sqrt{2 N}}\right)\left(1-P_{0, k}\right) \geq \mathbb{Q}\left(\frac{\lambda_{0, k}-N}{\sqrt{2 N}}\right) P_{0, k}+\mathbb{Q}\left(\frac{\lambda_{1, k}-N}{\sqrt{2 N}}\right)(1-$ $\left.P_{0, k}\right)=P_{f \text {,target }}$. Therefore, $\mathbb{Q}\left(\frac{\lambda_{0, k}-N}{\sqrt{2 N}}\right) \geq P_{f \text {,target }}$.

\section{Acknowledgements}

The authors would gratefully acknowledge the grants from the National Natural Science Foundation of China (61571250), the Natural Science Foundation of Ningbo City of China (2015 A610121), and the Natural Science Foundation of Fujian Province of China (2014J01243) and the K. C. Wong Magna Fund in Ningbo University.

\section{Authors' contributions}

$\mathrm{PL}, \mathrm{YH}$, and $\mathrm{CF}$ conceived and designed the study. $C F$ and $\mathrm{PL}$ performed the simulation experiments. CF and PL wrote the paper. YH, YL, MJ, and GW reviewed and edited the manuscript. All authors read and approved the final manuscript.

\section{Competing interests}

The authors declare that they have no competing interests.

\section{Publisher's Note}

Springer Nature remains neutral with regard to jurisdictional claims in published maps and institutional affiliations.

\section{Author details}

${ }^{1}$ College of Information Science and Engineering, Ningbo University, Ningbo, China. ${ }^{2}$ School of Information Science and Engineering, National Huaqiao University, Xiamen, China.

Received: 30 November 2016 Accepted: 3 June 2017

Published online: 28 June 2017

\section{References}

1. G Staple, K Werbach, The end of spectrum scarcity. IEEE Spectrum. 41(3), 48-52 (2004)

2. II Mitola, GQ Maguire, Cognitive radio: making software radios more personal. IEEE Pers Commun. 6(4), 13-18 (1999)

3. Y Pei, AT Hoang, Y-C Liang, in Proc. IEEE Int. Symp. Personal, Indoor and Mobile Radio Commun. (PIMRC 2007). Sensing-throughput tradeoff in cognitive radio networks: how frequently should spectrum sensing be carried out?, (2007), pp. 1-5

4. S Stotas, A Nallanathan, 2012, On the throughput and spectrum sensing enhancement of opportunistic spectrum access cognitive radio networks. Wireless Commun. IEEE Trans. 11(1), 97-107

5. J Zhang, et al, Sensing-energy efficiency tradeoff for cognitive radio networks. let Commun. 8(18), 3414-3423 (2014)

6. IEEE Standard for Information Technology Telecommunications and information exchange between systems Wireless Regional Area Networks
(WRAN) Specific requirements, Part 22: Cognitive Wireless RAN Medium Access Control (MAC) and Physical Layer (PHY) Specifications: Policies and Procedures for Operation in the TV Bands. IEEE std. 802.22a-2014

7. TYücek, H Arslan, A survey of spectrum sensing algorithms for cognitive radio applications. IEEE Commun. Surv. Tutorials. 11(1), 116-130 (2009)

8. Axell, Erik, et al, Spectrum sensing for cognitive radio:state-of-the-art and recent advances. IEEE Signal Process. Mag. 29(3), 101-116 (2012)

9. A Taherpour, M Nasiri-Kenari, S Gazor, Multiple antenna spectrum sensing in cognitive radios. IEEE Trans. Wireless Commun. 9(2), 814-823 (2010)

10. M López-Benítez, F Casadevall, Improved energy detection spectrum sensing for cognitive radio. let Commun. 6(8), 785-796 (2012)

11. J Wu, T Luo, G Yue, in Proc. IEEE 1st Int. Conf. Information Science and Engineering. An energy detection algorithm based on double-threshold in cognitive radio systems, (2009), pp. 493-496

12. SQ Liu, BJ Hu, XY Wang, Hierarchical cooperative spectrum sensing based on double thresholds energy detection. IEEE Communications Letters. 16(7), 1096-1099 (2012)

13. H Rifà-Pous, MJ Blasco, C Garrigues, Review of robust cooperative spectrum sensing techniques for cognitive radio networks. Wireless Personal Commun. 67(2), 175-198 (2012)

14. Y Saleem, MH Rehmani, Primary radio user activity models for cognitive radio networks: a survey. J. Netw. Comput. Appl. 43(1), 1-16 (2014)

15. SA Bayhan, Z Fatih, A Markovian approach for best-fit channel selection in cognitive radio networks. Ad Hoc Netw. 12(1), 165-177 (2014)

16. DJ Lee, WY Yeo, Channel availability analysis of spectrum handoff in cognitive radio networks. IEEE Commun. Lett. 19(3), 435-438 (2015)

17. M López-Benítez, F Casadevall, Empirical time-dimension model of spectrum use based on a discrete-time Markov chain with deterministic and stochastic duty cycle models. Veh. Technol. IEEE Trans. 60(6), 2519-2533 (2011)

18. S Geirhofer, L Tong, BM Sadler, Cognitive medium access: constraining interference based on experimental models. Selected Areas Commun. IEEE J. 26(1), 95-105 (2008)

19. MH Rehmani, et al, SURF: A distributed channel selection strategy for data dissemination in multi-hop cognitive radio networks. Comput. Commun. 36(10), 1172-1185 (2013)

20. N Moayeri, H Guo, in Proc. IEEE Symp. on New Frontiers in Dynamic spectrum. How often and how long should a cognitive radio sense the spectrum, (2010), pp. 1-10

21. Xing, Xiaoshuang, et al, Optimal spectrum sensing interval in cognitive radio networks. IEEE Trans. Parallel Distributed Syst. 25(9), 2408-2417 (2014)

22. K Haghighi, EG Strm, E Agrell, On optimum causal cognitive spectrum reutilization strategy. IEEE J. Selected Areas Commun. 30(10), 1911-1921 (2012)

23. J Heo, et al, Simultaneous sensing and transmission in cognitive radio. IEEE Trans. Wireless Commun. 13(4), 1948-1959 (2014)

24. KW Choi, E Hossain, Opportunistic access to spectrum holes between packet bursts: a learning-based approach. IEEE Trans. Wireless Commun 10(8), 2497-2509 (2011)

25. K Haghighi, EG Strom, E Agrell, Sensing or transmission: causal cognitive radio strategies with censorship. IEEE Trans. Wireless Commun. 13(6), 3031-3041 (2014)

26. $\mathrm{Xu}$, You, et al, Joint sensing period and transmission time optimization for energy-constrained cognitive radios. EURASIP J. Wireless Commun. Netw. 2010(1), 1-16 (2009)

27. PJ Green, Reversible jump Markov chain Monte Carlo computation and Bayesian model determination. Biometrika. 82(4), 711-732 (1995)

28. $Y$ Zeng, et al, A review on spectrum sensing for cognitive radio: challenges and solutions. EURASIP J. Adv. Signal Process. 2010(1), 1-15 (2010) 